https://doi.org/10.48009/1_iis_2005_53-58

\title{
A PROPOSED METHODOLOGY TO TEACH NETWORKING USING PORTABLE NETWORK PROGRAMMING PROJECTS
}

\author{
Dr. Ardian N. Greca, Georgia Southern University, agreca@georgiasouthern.edu \\ Dr. Sonny E. Butler, Georgia Southern University, esbutler@ georgiasouthern.edu \\ Dr. James K. Harris, Georgia Southern University, jkharris@georgiasouthern.edu
}

\begin{abstract}
The design of educational network programming software must be guided by educational theory. In this paper we present a framework for student-centered learning that is motivated by pedagogical theory. A set of three laboratory projects is demonstrated together with their assessment and evaluation principles.
\end{abstract}

Keywords: Data networking, portable network configuration, network applications, teaching methodology

\section{INTRODUCTION: MOTIVATION AND GOAL(S)}

Current pedagogical research shows that students learn best by manipulating new concepts whether verbally, visually, or manually $[1,2,3]$. Research also confirms that learning is nonlinear, recursive, complex, relational, and natural in humans and has shown that learning is enhanced in contexts where learners have supportive relationships, a sense of ownership and control over the learning process, and can learn with and from each other in safe and trusting learning environments.

In the learning process students pass through three levels of learning:

1. knowledge

2. skills and accomplishment

3. expertise

Knowledge. Most teaching methodologies are designed to promote knowledge/information transfer. While knowledge transfer is a necessary component of learning, it is not sufficient by itself to change learning behavior.

Skills and accomplishment: If the objective of learning is to promote skills and develop proficiency in performing a particular task, it is important to simulate the task so a student can follow the steps in the appropriate order and practice. At this point however, students still have not learned how to adapt and reapply these skills in different contexts.

Expertise: To deliver the technological skills that most companies want, the learning process must integrate the aspects of where, when, and why the skill is used in a real world environment. The best tools to provide this are found in expert instruction combined with practice in a realworld context.

The rapid growth of network applications and the huge amount of information available requires new approaches for learning in networking courses. The conventional approach of having an instructor prepare lessons and explain topics is no longer effective and cannot possibly be used to 
convey the necessary concepts. Expected objectives and outcomes prepared by the instructor alone do not sufficiently involve students in the learning process.

For our upper division courses in data communications and networking, and distributed web systems design we have developed a set of three projects to help students better understand concepts, receive immediate feedback on their comprehension and enhance the learning process. The goal is to develop network programming projects that contribute to rapid feedback and learning and create an effective learning environment in the classroom by going through three levels of learning. The main objectives are:

- To understand basics of LANs, topologies, and wireless technologies through hands-on construction;

- To show how to set up a portable closed network that can be used for many types of network projects;

- To provide an understanding of the client server paradigm, distributed system operation, and application layer programming through the creation of client-server programs at both the socket and application levels and

- To promote a highly interactive shared learning environment.

The results obtained so far using this method have been promising. The remainder of this paper is organized as follows.

- Introduction of the project(s)

- Assessment and evaluation principles

- Conclusions and future work.

\section{LABORATORY PROJECTS}

\section{Wireless network configuration}

The first project consists of setting up a network in the classroom for students to work in. For this project students need laptop computers with wireless Internet cards (plug-in card PCMCIA or incorporated in the hardware), an access point, that in our case will be a wireless router. Students are then asked to set up and configure the network as shown in Fig 1.

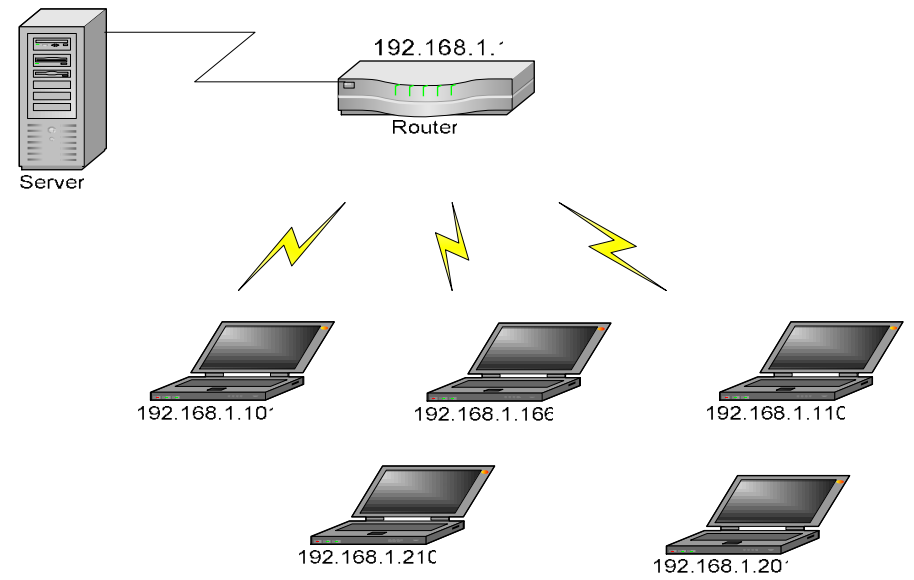

Figure 1. Network configuration. 
The router generates IP addresses for each computer connected to the network. Like other LAN technologies, the wireless LANs use sharing, i.e., all computers participating are configured to the same radio frequency, and therefore must take turns sending packets. The wireless LANs use the modified scheme known as Carrier Sense Multiple Access With Collision Avoidance. In order to perform this project, students are asked to work in groups and define their own objectives for the group. Some of the objectives submitted from students include, understanding network topologies, wireless technologies, wireless protocols, network configuration, DHCP configuration, DNS, etc. They then are required to follow their stated objectives and perform the project. The role of the instructor will be to guide them and discuss any problem(s) that might arise.

\section{Network application: Chat}

The second project consists of programming a Windows based chat application. Although networks transfer data from one point to another, the network itself neither generates nor understands the data being sent. All data processing is carried out by application programs. Applications follow the client/server paradigm when they communicate. The server application starts first and waits for contact from a client. The client contacts the server by specifying its location and requesting communication. The client and server then begin exchanging data. After they finish sending data, both the client and server terminate communication. At Georgia Southern University, we have programmed this application using the $\mathrm{C}++$ language. The flowcharts showing the server and client sending a single request and receiving a single reply, are displayed in Fig. 2.

Server

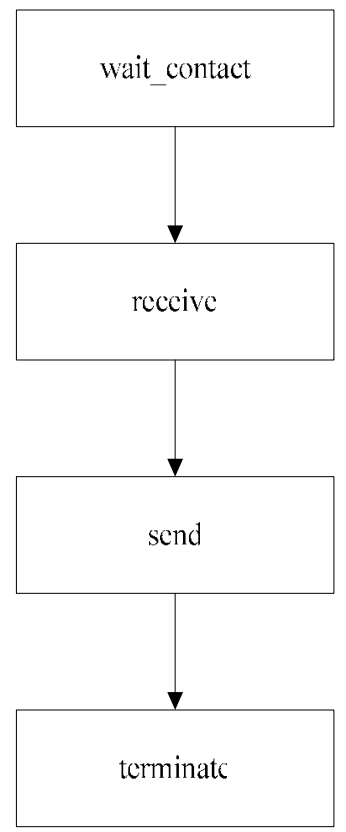

Client

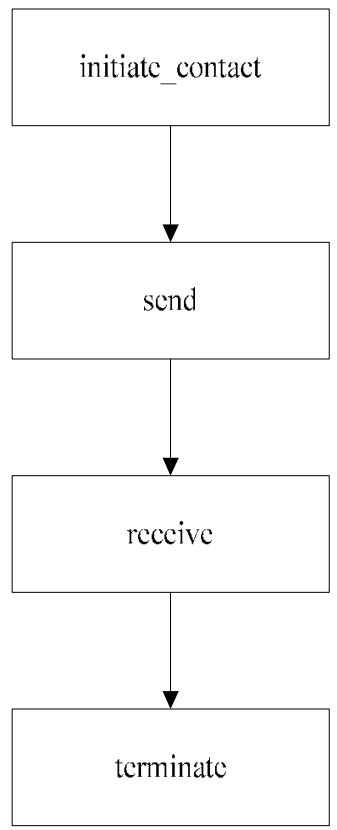

Figure 2. The client/server paradigm. 
For this project students are required to prepare and follow their objectives to complete the chat application. The requirements submitted from students for understanding of the client-server paradigm include:

- socket programming,

- operating system requirements,

- multithreading

In order to complete the application, students work in groups and each group demonstrates their application to the instructor. Upon completion, students are required to use their programs and chat with their peers. The role of the instructor is to guide and discuss problems and solutions with the different groups.

\section{Network application for online video transmission}

The third project is a web application that allows transmission of real time video using a webcamera. Code is provided that captures the video signal from a web-camera and stores the images on the client's computer. Students are shown how to post the images on their own web page located on a central server. Different groups can use the "chat" application to interact while constructing their web pages allowing participants to interactively share in their learning experiences thereby increasing participation and the exchange of information. When finished with the third project, students can then use both the chat application and live-video feed to communicate with other groups. Since pages are published on a central server, they are available to all groups. The objectives set by students include understanding application protocols, their relation to the underlying network protocols, etc.

\section{ASSESSMENT AND EVALUATION PRINCIPLES}

While designing these projects, instructors have tried to identify problems suitable for students and related to their environment so as to present authentic real world scenarios. Some of the basic ideas in this process are to give students the responsibility for defining their learning objectives and experience the planning necessary to solve the problem(s) and to encourage collaborative learning among the teams. Their learning experience is based on the pedagogy defined earlier as they pass through all three levels of learning.

Measuring successful outcomes of the learning process is an important issue. Instructors have considered the following questions important for evaluating success.

What changes in the teaching process can be observed that enhance learning? Teaching networking courses in the CS curriculum poses some special challenges. The main one is that the field is so broad and students will "get by" by only following the teachers instructions. The course must be refined and organized for effective learning. To obtain this goal, students should go through all three levels of learning.

Research shows that grade point average is one of the best indicators of success in a course. Table 1 summarizes the GPA class average for courses with and without a set of projects. Both terms with projects show an increase in student performance. Note that the set and number of 
projects are different for Spring 2003 and Spring 2005. Also, the course organization is different in Spring 2005 compared with those of the previous terms.

Table 1. Average student's GPA for different terms with/without set of projects

\begin{tabular}{ccccc} 
Term & $\begin{array}{c}\text { Number } \\
\text { of } \\
\text { students }\end{array}$ & $\begin{array}{c}\text { Average GPA } \\
\text { without projects }\end{array}$ & $\begin{array}{c}\text { Average GPA with } \\
\text { a different set of } \\
\text { projects }\end{array}$ & $\begin{array}{c}\text { Average GPA with } \\
\text { set of projects } \\
\text { presented in this } \\
\text { paper }\end{array}$ \\
\hline Spring 2002 & 14 & 2.218 & - & - \\
Spring 2003 & 28 & - & 2.75 & - \\
Spring 2005 & 18 & - & - & 3.04
\end{tabular}

What changes in learning process can be observed that create new learning partnerships, collaboration and communities? By introducing students to these projects, we not only expect to enhance the learning, but also intend to organize the course in the way that will enable students to obtain qualities most wanted by employers. Such qualities include communications skills, team-work skills, motivation/initiative, honesty, interpersonal skills, analytical skills and selfconfidence.

How do students use knowledge and complex reasoning compared to the recall of low-level information? In order to succeed and reach the objectives set for this course, instructors should be interested and concerned about students. This interest starts with discussions and other interactions with students. Having extra office hours for students allows them to come and present their progress also express their interests. Beside these standard opportunities to increase student-teacher interaction, other methods such as anonymous surveys were used. Some of the positive student feedback included the following:

- "After this project it is easy for me to see why it is an access point that will let multiple computer utilize the ports to access the bandwidth given on the internet."

- "I can honestly say I was kind of a novice to the whole idea of an IP. I felt that I knew I had one and it singled me out on the internet."

- "The project gave us some hands-on experience with real networking equipment and problems that arise when trying to setup a network."

- "The project was very educative experience. I can better understand the application protocols now."

- "I learned a lot from this project. I saw how all the pieces of the network that we had discussed in class actually interacted with each other and how their function was a key to the network's operation."

Some of the concerns, which students expressed, included the following:

- "Label the router and computers so everyone can easily distinguish the computer they are communicating to."

- "More time is required on performing some projects."

- "I did not understand at all how to configure the wireless connection." 
Based on our results so far, we strongly believe that these projects are worthwhile in helping students to understand the concepts and learn the art and science of data communications.

\section{CONCLUSIONS AND FUTURE WORK}

There are a number of reasons instructors believe this type of learning environment will enhance students' learning and increase the feedback. Students and instructors are involved in creating the learning objectives and the learning environment which allows everyone to participate. Students gain the knowledge on how to apply the subject content to their work environment and to personal applications. They feel more comfortable in this learning environment and will be able to use supporting materials in an easy-to-use, self-directed format, etc. This learning method can be applied in areas other than academia. A good area for utilizing techniques of this nature would be in the private sectors of industry. Students that are involved in the learning process are better prepared for the future challenges. This opens many opportunities for additional projects at different levels of difficulty depending upon the instructors and students. Projects of this nature are portable and can be performed on microcomputers using small wireless local area networks.

\section{REFERENCES}

1. Cohen, M. L., Grady, M. \& Springer, S. (December 2003). Talk About Teaching and Learning, Almanac, 50(9). (http://www.upenn.edu/almanac/v50/n09/teaching.html)

2. APA, Learner-Centered Psychological Principles: A Framework for School Redesign and Reform, November 1997. (http://www.apa.org/ed/lcp.html)

3. Schlag, P. V. (July 2002). Global Knowledge Surrounds Learning technology offers LearnerCentered Training, Learning \& Training Innovations Magazine. (http://www.ltimagazine.com)

4. Soloway, E. Jackson, S. L., Klein, J., Quintana, C., Reed, J., Spitulnik, J., Stratford, S. J., Studer, S. Jul, S. Eng, J., \& Scala, N. (1996). Learning Theory in Practice: Case Studies of Learner-Centered Design. (http://www.acm.org/sigchi/chi96/proceedings/papers/Soloway/es_txt.htm)

5. Greca, A. N., Cook, R. P., \& Harris, J. K. (January 2004). Enhancing Learning in a Data Communication and Networking Course with Laboratory Experiments, The Journal of Computing Scienes in Colleges, 19(3), pp 79-88.

6. Tanenbaum, A. S. (2003). Computer Networks, $4^{\text {th }}$ Ed., Prentice Hall.

7. American Association for the Advancement of Science [AAAS], Project 2061. (1993). Benchmarks for science literacy. New York: Oxford University Press.

8. McCombs, B. L. The Learner-Centered Framework on Teaching and Learning As a Foundation for Electronically Networked Communities and Cultures. (http://www.pt3.org/VQ/html/mccombs.html) 